\title{
CLIMA Y SATISFACCIÓN LABORAL COMO PREDICTORES DEL DESEMPEÑO: EN UNA ORGANIZACIÓN ESTATAL CHILENA
}

\section{ORGANIZATIONAL CLIMATE AND JOB SATISFACTION AS PERFORMANCE PREDICTORS IN A CHILEAN PUBLIC ORGANIZATION}

Recibido: 02 de Febrero del 2011 | Aceptado: 30 de Agosto del 2011

\author{
ANDRÉS A. RODRÍGUEZ M. ; MARIA PAZ RETAMAL J JOSÉ N. LIZANA $_{3}$; FELIPE A. CORNEJO
} (UNIVERSIDAD ANDRÉS BELLO, Santiago de Chile, CHILE)*

\begin{abstract}
RESUMEN
Actualmente existe consenso respecto a que el clima organizacional y la satisfacción laboral son variables fundamentales dentro de la gestión de las organizaciones. Sin embargo, no está claro cuáles son los efectos específicos que tienen dichas variables sobre el desempeño laboral en general, ni tampoco sobre los aspectos específicos del desempeño, tales como el comportamiento normativo, la productividad y las relaciones sociales.

El objetivo de este estudio es determinar si el clima organizacional y la satisfacción laboral son predictores significativos tanto del desempeño laboral como de sus dimensiones específicas. Se aplicaron los cuestionarios de clima organizacional de Litwing y Stringer (1989) y de satisfacción laboral JDI y JIG (1969) a un total de 96 trabajadores de un organismo público de la Región del Maule. Los cuestionarios fueron contestados de forma individual y en un solo momento. Esta aplicación fue realizada junto al proceso de evaluación anual de desempeño de la institución. En esta evaluación se aplicó un cuestionario confeccionado por la institución cuyo objetivo era evaluar tres dimensiones del desempeño de los funcionarios ("comportamiento funcionario, condiciones personales, rendimiento y productividad).

Se evidencia que existe una relación significativa entre el clima, la satisfacción y el desempeño. Y que el desempeño es predicho de mejor forma por las variables en conjunto. Considerando las dimensiones del desempeño, sólo el clima predice significativamente el comportamiento funcionario y las condiciones personales, mientras que la satisfacción sólo predice el Rendimiento y la Productividad.
\end{abstract}

PALABRAS CLAVE: Clima organizacional, satisfacción laboral, desempeño.

\section{ABSTRACT}

Nowadays, there is a general agreement on the idea that organizational climate and job satisfaction are strongly associated as fundamental variables on organization management. Hence, there is not a clear vision with respect on how these variables are related with the job performance.

The purpose of this study is to determine if exists a significant relation between the variables and if climate organizational or job satisfaction are significant predictors of the job performance. The study is sectional correlational, using quantitative (instruments) and qualitative (one focus group) techniques, in 96 workers from public institution of Maule's region. The results showed that exist a significant relation between the variables and that the job performance is better by both variables. Considering the dimensions of job performance, only the climate predict significantly the "Worker Behavior" and "Personal Conditions", by the other hand the satisfaction predict significantly Efficiency and Productivity.

KEY WORDS: Organizational climate, job satisfaction, job performance.

\footnotetext{
1. Psicólogo del Liceo Santa Marta Talca, encargado de diagnóstico y evaluación psicológica enseñanza media. Email: arodmen@gmail.com 2. Psicóloga del Instituto Profesional Iplacex, Talca, Chile. Email: mretamal@iplacex.cl

3. Master of Business Administration, Facultad de Ciencias Sociales, Universidad de Andrés Bello. Email: joselizanal@gmail.com

4. Alumno del Magister de Gestión Tecnológica. Email: felipecornejot@gmail.com

* La universidad de referencia es filiación académica exclusiva del autor José N. Lizana.
} 


\section{INTRODUCCIÓN}

En el último tiempo, la gestión de recursos humanos (RR.HH.) ha tomado gran importancia para las organizaciones, en especial para los servicios públicos, pues permite generar ventajas comparativas asociadas al mejor desempeño de las tareas y servicios generados dentro de ellas. Conjuntamente, se ha concientizado y valorado la importancia de establecer mejores condiciones laborales y potenciar la calidad de vida de las personas (Sziklai, 2006). En este contexto, la generación de investigaciones que consideren variables como el clima organizacional, la satisfacción laboral o el desempeño toman especial relevancia, pues contribuyen de forma transversal a la gestión de RR.HH. y al bienestar de las personas, ya sea por medio de la detección de necesidades de capacitación, la formulación de programas de promoción de la calidad de vida laboral y de la salud ocupacional, el diseño de sistemas de gestión del capital humano y el fortalecimiento de políticas de desarrollo de personas (Imran, Saeed, Anis-Ul-Haq \& Fatima, 2010).

En consultoría, una de las actividades más demandadas corresponde a los estudios de clima organizacional. Sin embargo, la precisión o conocimiento exacto sobre los aportes que este tipo de estudios tiene, no es completamente clara. Lo que genera, en muchas ocasiones, resultados estériles y que poco aportan al desarrollo de las organizaciones (Gómez, 2004; Silva, 1996). En este contexto resulta cada vez más necesario el desarrollo de procesos diagnósticos precisos que permitan identificar las condiciones del clima organizacional que influyen significativamente sobre el bienestar de los trabajadores.

Para Silva (1996), muchas veces las organizaciones ponen en marcha programas de intervención 0 capacitaciones, $\sin$ analizar convenientemente todos los elementos importantes que deben ser mejorados, pasando por alto la historia de la organización así como los modelos que dan cuenta de su complejidad. Como consecuencia, dichos programas fracasan 0 tienen un impacto sólo a corto plazo.

A pesar del gran número de investigaciones existentes sobre clima y satisfacción (Carr, Schmidt, Ford, DeShon, 2003; Cuadra, Veloso, 2007; Chiang, 2004; Chiang, 2008), muy pocos son los esfuerzos que se centren por clarificar los efectos diferenciales que estas variables tienen sobre el desempeño de las personas. Para clarificar los efectos y algunas diferencias se plantean aquí algunos antecedentes teóricos de las dimensiones clima y satisfacción laboral, para luego abordar la relación que éstas tienen con el desempeño de las personas.

Clima, satisfacción laboral y desempeño: Algunas clarificaciones conceptuales.

En términos conceptuales existen muchas definiciones del concepto de clima laboral u organizacional (Peiró, 1985; Salgado, Remeiseiro \& Iglesias, 1996; Furnham, 2001; Boada, Vallejos \& Agulló, 2004; Peraza \& García, 2004; Rodríguez, 2004; Patterson, et al., 2005; Reza \& Jahandari, 2005; Martin \& Cullen, 2006); muchas de estas definiciones derivan del enfoque teórico que presenten los autores respecto al concepto (clima como variable dependiente, independiente, mediadora, etc). Para la presente investigación se ha tomado la definición de Litwin \& Stringer (1968), la cual señala que el clima organizacional es una propiedad del ambiente organizacional descrita por sus miembros. En este sentido, el clima organizacional se origina producto de efectos subjetivos percibidos por los trabajadores respecto del sistema formal en que se desenvuelven, del estilo informal de los administradores y de factores organizacionales (características del trabajo, condiciones del empleo, etc.). Estos 
factores, afectarían las actitudes, creencias, valores y motivación de las personas.

Respecto a la satisfacción laboral, esta ha sido definida de múltiples formas, siendo considerada inicialmente como un estado emocional 0 afectivo de los individuos hacia su trabajo (Davis \& Newstrom, 1999), también ha sido definida como cogniciones o evaluaciones perceptuales de un individuo hacia su trabajo (Alfaro \& Vecino, 1999). Mientras que hoy en día, la satisfacción laboral ha sido considerada como una actitud 0 conjunto de actitudes generalizadas hacia el trabajo (Peiró, 1985; Gamero, 2003; Alcover, Martínez \& Rodríguez, 2004).

En cuanto al desempeño organizacional, no existe consenso entre los autores respecto a los factores que involucra dicha variable (Salgado, 2006). Tradicionalmente ha sido relacionado con dimensiones financieras, con el posicionamiento $y$ adaptación a los cambios en el mercado, con los márgenes promedios de ganancia organizacional, con el rendimiento en base al capital empleado, con el crecimiento en las ventas, etc. (Kangis \& Williams, 2000). Asimismo, hay quienes lo consideran en base a metas logradas y recursos que se utilizan para lograrlas (De Juan, 2004), y quienes centran la atención en la inestabilidad económica (Fey \& Beamish, 2001). Otros han estudiado el desempeño a partir de indicadores de eficiencia y eficacia, o considerando índices de absentismo y rotación en la organización (Brutus \& Gorriti, 2005). Sin embargo, pareciese ser que la aproximación económica del desempeño es la visión predominante hasta el día de hoy, dejando poco espacio para su medición a partir de variables más subjetivas (Patterson, Warr \& West, 2004).

En el contexto de las instituciones públicas el desempeño exitoso de una tarea no se encuentra determinado única $y$ exclusivamente por factores económicos, sino más bien, en muchas ocasiones se relaciona con aspectos gubernamentales, sociales y políticos propios de la función del estado. Por tales motivos la aproximación hacia el estudio del desempeño en organismos públicos debe considerar una visión integral, asociada a la conducta, el cumplimiento de normas y el logro de los objetivos individuales e institucionales (Chiang, Salazar, Huerta \& Núñez, 2008). Por tales motivos el estudio del desempeño en organizaciones públicas debiese considerar como medida el "qué tan bien" ejecuta una persona su rol en el trabajo. Este rol puede medirse como un índice de resultado (productividad) o comparando las conductas de las personas frente a las expectativas de la organización, como es el caso de la evaluación de desempeño profesional (Pritchard, 1992). Pareciese ser que en el caso de las instituciones públicas no basta con considerar únicamente los factores asociados a la productividad, sino más bien debe considerarse una medida de desempeño que integre aspectos normativos, sociales y profesionales (Carr, Schmidt, Ford \& DeShon, 2003).

Relación entre clima organizacional satisfacción laboral.

Si bien la relación entre clima y satisfacción es clara, el amplio espectro de definiciones de ambas variables ha limitado la profundidad de su análisis, encontrando escasos estudios que clarifiquen la forma en que se manifiesta esta relación (Chiang, 2004; Chiang, 2008, Remeseiro \& Iglesias, 1996; Silva, 1996). De esta forma, la investigación se ha enfocado en el estudio de la relación mediante análisis de correlaciones, encontrando que existe una relación significativa y positiva entre el clima organizacional y la satisfacción laboral, de manera tal que a mejor clima percibido más satisfechos estarán las personas (Salinas, Laguna \& Mendoza, 1994; Salgado, Remeseiro \& Iglesias, 1996; Silva, 1996; Parker et al., 2003; Chiang, 2004; Patterson, Warr \& West, 2004; Griffith, 2006; Schulte, Ostroff \& Kinicki, 2006). Cabe destacar que las relaciones encontradas en su mayoría 
son de tamaño moderado (correlaciones entre 0,5 y 0,7 ).

Hoy en día, los autores coinciden en señalar que ambos constructos son independientes entre sí y que el constructo de clima organizacional es descriptivo, es decir, una descripción que hacen los miembros de su organización. Mientras que la satisfacción laboral obedece a una evaluación que hace un individuo de su trabajo, teniendo un componente afectivo (Silva, 1996). A pesar de la evidente relación entre clima y satisfacción, pareciese ser que no todos los componentes que articulan a ambas variables comparten de igual forma dicha relación. Schratz (1993), a través de un meta-análisis encontró que existen dimensiones de clima organizacional y satisfacción laboral que se han relacionado más históricamente entre sí. Las dimensiones de clima organizacional que más se han relacionado con la satisfacción laboral son el apoyo de la supervisión, las recompensas, la responsabilidad (autonomía individual en el trabajo), las relaciones interpersonales (calidez) y las competencias del empleado. Las dimensiones de satisfacción laboral que más se han relacionado con el clima organizacional son la satisfacción con el sueldo, la supervisión, los compañeros y el ascenso.

Relación entre clima organizacional y desempeño organizacional.

Respecto a la relación particular entre clima y desempeño, Silva (1996), establece que hablar de la influencia del clima sobre el desempeño implica considerar tanto las repercusiones del clima sobre las actitudes y comportamientos, como en el desempeño organizacional, ya que en las percepciones que forman los individuos de su ambiente de trabajo juegan un papel importante la manera en que está estructurada la tarea, el sistema de recompensas, el modo en que las decisiones se controlan, el tipo de comunicación, etc. Así han surgido numerosos modelos teóricos que buscan graficar la mediación de las actitudes en el trabajo, en especial la satisfacción laboral y su relación con el clima y el desempeño (Kopelman, Brief \& Guzzo, 1990; Parker et al., 2003). Desde esta visión la satisfacción laboral debiera tener un efecto más directo sobre el desempeño mientras que el clima tendría un efecto más bien indirecto, mediado por las actitudes.

Los autores suelen ser cautos cuando se trata de expresar el valor predictivo que el clima tiene sobre el desempeño. Por ejemplo, Silva (1996), señala que el clima es simplemente una herramienta útil para comprender y mejorar el desempeño, por lo que los autores hablan más bien del impacto potencial del clima sobre el desempeño como un determinante indirecto de éste, sin establecer así una relación causal entre ambas variables (Silva, 1996; Burton \& Lauridsen, 2004). Algunos metaanálisis al respecto, han revelado que dentro de las dimensiones de clima que más se han relacionado con el desempeño y el rendimiento de las personas son los estilos de supervisión, el apoyo, el riesgo y la toma de decisiones, las recompensas, las relaciones entre compañeros y la estructura (Schratz, 1993).

\section{Relación entre satisfacción laboral y desempeño organizacional.}

Los autores señalan que la relación entre la satisfacción laboral y el desempeño es más clara y directa que la relación entre el clima y el desempeño, siendo la satisfacción un importante predictor del desempeño (Kopelman, Brief \& Guzzo, 1990; Parker et al., 2003, Patterson, Warr \& West, 2004; Griffith, 2006). Se ha encontrado una relación significativa y negativa entre la satisfacción laboral y aspectos negativos del comportamiento organizacional tales como el abstentismo y la rotación en el trabajo de modo que quienes están más insatisfechos presentan mayores tasas de absentismo y 
rotación. Igualmente han encontrado una relación positiva con el rendimiento y la productividad, de modo que quienes están satisfechos con su trabajo presentan un mejor rendimiento y son más productivos (Saari \& Judge, 2004). Así, Patterson, Warr \& West (2004), señalan que la satisfacción laboral se ha relacionado más con el desempeño, cuando éste es medido a través de aspectos económicos más bien relacionados con la productividad.. Salinas, Laguna \& Mendoza (1994), señalan que los modelos de satisfacción como por ejemplo, el modelo de Vroom, enfatizan en que los individuos están satisfechos con su trabajo hasta el grado en que les permita obtener lo que desean, y su desempeño será eficiente cuando los conduzca a la satisfacción de sus necesidades.

Relación entre clima organizacional, satisfacción laboral y desempeño organizacional.

Los primeros modelos que consideran estas tres variables en conjunto son el modelo de Litwin \& Stringer (1968) y el de Lawler, Hall \& Oldman (1974), en ambos casos se señala que las variables de estructura organizacional y de procedimientos organizacionales, tendrían un efecto directo sobre el clima organizacional, el cual afectaría la motivación, y las conductas y resultados organizacionales, entre los cuales se encontraría la satisfacción laboral y el desempeño. Estas últimas variables tendrían una relación indirecta con las variables estructurales y procesales. Sin embargo algunos modelos basados en la evidencia empírica como el de Kopelman, Brief \& Guzzo (1990), señalan que la satisfacción laboral media la relación entre clima y desempeño, congruentemente Parker et al. (2003) encontró a partir de una investigación meta-analítica de 121 muestras que las actitudes hacia el trabajo, especialmente la satisfacción laboral y la motivación median la relación entre el clima y el desempeño. Existe evidencia contradictoria sobre la relación entre las tres variables, por ejemplo Patterson, Warr \& West (2004), encontraron en una investigación de 42 industrias que el clima organizacional influye en el desempeño a través de la satisfacción, la que puede utilizarse como predictor de la productividad, siendo la satisfacción laboral un mediador en la relación clima y desempeño. Sin embargo, Griffith (2006) no encontró tal resultado en su evaluación de una institución educativa de carácter público. Como se ha dicho, la mayoría de los teóricos han predicho que las reacciones afectivas mediarían la relación entre el clima y el desempeño, por lo tanto la satisfacción laboral al ser de connotación más bien afectiva y una actitud, debiera ser un mediador de la relación entre el clima y desempeño, y por ende tener un efecto más directo sobre el desempeño que el clima. Este último, por lo tanto tendría un efecto más bien indirecto en el desempeño. Así, la satisfacción debiera ser un mejor predictor del desempeño que el clima. Sin embargo aquellas investigaciones que han enfatizado en la capacidad predictiva de la satisfacción sobre el desempeño, se han centrado en indicadores de desempeño más bien centrados en ámbitos de productividad y rendimiento (Kopelman, Brief \& Guzzo, 1990; Parker et al., 2003; Patterson, Warr \& West, 2004), sin considerar otros elementos tales como el complimiento de las normas dentro de la organización o la conducta del individuo dentro de la organización. Estos aspectos resultan relevantes para la evaluación del desempeño profesional dentro de organizaciones públicas puesto a que en este tipo de organizaciones el rendimiento y la productividad no son los únicos elementos a considerar dentro de la evaluación de desempeño y por ende la hipótesis respecto al rol mediador de la satisfacción sobre el desempeño podría no ser $\tan$ evidente en este tipo de evaluaciones (Chiang, Salazar, Huerta \& Núñez, 2008). Si se considera que gran parte de las investigaciones y diagnósticos desarrollados para evaluar variables como clima y satisfacción, por lo general se desarrollan dentro del contexto de intervenciones y consultorías que en 
escasas ocasiones tienen difusión masiva. La información científica que caracterice cómo el clima y la satisfacción se relacionan para predecir el desempeño se hace escasa. Si más aún, se tiene en cuenta la ambigüedad de la medida desempeño, y la moderada evidencia relativa a la medición del clima y la satisfacción en organizaciones públicas, resulta relevante verificar $y$ clarificar la relación de estas variables respecto al concepto de desempeño propio de las organizaciones públicas, que ayude a identificar un efecto diferencial que pueda tener el clima y la satisfacción como predictores de los distintos aspectos del desempeño.

\section{OBJETIVOS}

\section{Objetivo General}

Identificar la relación entre clima organizacional, satisfacción laboral y desempeño en trabajadores de una organización pública chilena.

\section{Objetivos específicos}

- Determinar si existe relación entre las dimensiones de clima organizacional y la satisfacción laboral.

- Determinar si existe relación entre las dimensiones de clima organizacional y el desempeño.

- Identificar si existe relación entre las dimensiones de satisfacción laboral y el desempeño organizacional.

- Determinar si la satisfacción laboral es un mejor predictor del desempeño organizacional que el clima organizacional.

- Determinar si el clima organizacional y la satisfacción laboral son predictores significativos de las dimensiones específicas del Desempeño.

\section{HIPÓTESIS}

H1: Existe una relación significativa entre dimensiones de Clima Organizacional y Satisfacción Laboral.

H2: Existe una relación significativa entre dimensiones de Clima Organizacional y Desempeño Organizacional.

H3: Existe una relación significativa entre dimensiones de Satisfacción Laboral y Desempeño Organizacional.

H4: La Satisfacción laboral es mejor predictor del Desempeño Organizacional, que el Clima Organizacional.

\section{MÉTODO}

Se realizó un estudio cuantitativo. El diseño de estudio fue no experimental, transversal y correlacional.

Muestra

Se realizó un muestreo no probabilístico por conveniencia para seleccionar un total de 96 trabajadores de una organización pública de la Región del Maule perteneciente al sector agrícola, para la realización del estudio se consideraron todas las oficinas existentes en la región (dos de ellas ubicadas en Talca, una en Curicó, una en Linares, una en Cauquenes y una en Parral). La evaluación tuvo en cuenta a la totalidad del personal, considerando todos los niveles jerárquicos (jefes de oficina, profesionales técnicos, supervisores, profesionales de apoyo, administrativos y secretarios). Del total de participantes 54 eran hombres $(56.3 \%)$ y 42 eran mujeres $(43.7 \%)$. El promedio de edad es de 41.5 años.

\section{Instrumentos.}

-OCQ de Litwin y Stringer para medir clima organizacional: este instrumento posee 50 ítems y mide 9 dimensiones del clima (estructura, recompensas, responsabilidad, 
calidez, apoyo, conflicto, identidad, normas, y riesgo). Además, es posible obtener un índice de clima general. La confiabilidad por consistencia interna de este instrumento obtenida en esta investigación es buena ( $a$ $=0.855$ ).

-Cuestionario de Satisfacción por facetas (JDI) de Smith, Kendall \& Hulin (1969): validado preliminarmente para una muestra maulina por Castro \& Paredes (2006), este instrumento mide satisfacción laboral y posee 72 ítems, mide 5 dimensiones (satisfacción con el trabajo, con la supervisión, con los compañeros de trabajo, con el sueldo y con las oportunidades de ascenso). La confiabilidad de este instrumento obtenida en esta investigación es muy buena $(\alpha=0.925)$.

-Cuestionario de Satisfacción General (JIG) creado por Iroson, Brannick, Smith, Gibson \& Paul (1989): validado para una muestra maulina por Castro \& Paredes (2006), mide la satisfacción con el trabajo en general y posee 18 ítems. La confiabilidad de este instrumento obtenida en esta investigación es buena $(a=0.880)$.

-Cuestionario Institucional de Evaluación de Desempeño: diseñado específicamente para la organización por el ministerio a cargo de dicha institución. Mide 3 dimensiones: "Comportamiento Funcionario" (conducta de los individuos en cuanto al cumplimiento de las normas y obligaciones de trabajo), "Condiciones Personales" (mide cómo se comportan los individuos en sus relaciones interpersonales en el trabajo y como se comunican con los demás), y "Rendimiento y Productividad" (mide el cumplimiento de metas y tareas de trabajo en base a los recursos asignados a los trabajadores para cumplir tales tareas).

\section{Procedimiento}

Se solicitó el consentimiento de la organización para aplicar los instrumentos de medición, los cuales fueron entregados a los participantes pidiéndoles su apoyo voluntario y explicándoles los objetivos de la investigación. Posteriormente, se solicitó a la Encargada de Recursos Humanos los informes de evaluación de desempeño de los funcionarios (contando con la previa autorización del director regional).

\section{Plan de análisis}

Para el análisis de los datos se utilizó el programa estadístico SPSS 15.0 para Windows. Para identificar la relación entre satisfacción y clima se realizaron correlaciones de Pearson. Con el objetivo de determinar si las potenciales variables independientes (clima y satisfacción), predicen significativamente a la dependiente (desempeño), así como identificar qué variable tiene mayor poder predictivo se realizaron Regresiones Lineal Múltiples, empleando modelos stepwise (paso a paso).

\section{RESULTADOS}

Relación entre clima organizacional y satisfacción laboral.

La correlación entre el clima organizacional general y la satisfacción laboral resultó estadísticamente significativa, presentando una relación positiva y una asociación de un $41.3 \%(r=0.413 ; p<0.05)$.

Considerando las dimensiones de satisfacción laboral, todas éstas obtuvieron una correlación significativa y positiva con el clima general ( $p s<0.05)$. La dimensión que resultó más asociada al clima organizacional general es Tarea en el Trabajo Actual $(r=0.477)$, luego siguen Supervisión $(r=0.452)$, Compañeros de Trabajo $(r=0.402)$, Oportunidades de Ascenso $(r=0.344)$ y Sueldo $(r=0.303)$.

Considerando las dimensiones del clima organizacional, 7 de éstas correlacionan significativa y positivamente con la satisfacción general $(p s<0.05)$, éstas son: Identidad $\quad(r=0.393)$, Calidez $(r=0.374)$, Apoyo ( $r=.336)$, Responsabilidad $(r=0.261)$, 
Normas $(r=0.258)$, Recompensa $(r=0.256)$ y Estructura $(r=0.254)$.

\section{Relación con desempeño organizacional}

El clima organizacional correlacionó significativa y positivamente con el desempeño general $(r=0.539 ; p<0.05)$. A su vez, considerando las dimensiones del clima organizacional, el desempeño correlaciona significativamente con 8 de ellas (ps<0.05), éstas son: Identidad $(r=0.510)$, luego viene Recompensa $(r=0.459)$, Apoyo $(r=0.452)$, Calidez $\quad(r=0.429)$, Normas $\quad(r=0.323)$, Conflicto $(r=0.302)$, Estructura $(r=0.290)$ y Riesgo $(r=0.218)$.

El desempeño general correlacionó significativa y positivamente con la satisfacción general $(r=0.466)$ y con 4 de las dimensiones de ésta $(p s<0.05)$ : Tarea en el Trabajo Actual $(r=0.425)$; Compañeros de Trabajo $(r=0.338)$; Supervisión $(r=0.281)$ y Sueldo $(r=0.213)$.
Satisfacción y el clima como predictores del desempeño

El análisis de regresión lineal identificó dos modelos significativos, uno con solo el clima como predictor del desempeño y otro con la satisfacción y el clima como predictores del desempeño en conjunto. Ambos modelos resultaron significativos, en el que caso del modelo que considera al clima y la satisfacción en conjunto como predictores del desempeño, se explica un $38,4 \%$ de su variabilidad $(F(2 ; 93)=26.334 ; \quad p<0.05)$, mientras que el clima por sí solo explica el $28.2 \%$ de la variabilidad del desempeño $(F(1 ; 94)=38.401 ; p<0.05)$. Al analizar los coeficientes de regresión del modelo en conjunto, se confirma la tendencia, donde el clima tiene mayor poder como predictor del desempeño $(\beta=0.417 ; \quad t(95)=4.587$; $p<0.05)$. Mientras que el poder predictivo de la satisfacción es menor $(\beta=0.294 ; t(95)=$ $3,228 ; p<0.05)$.

TABLA 1.

Coeficientes de la satisfacción y el clima general como predictores del desempeño.

\begin{tabular}{|c|c|c|c|c|c|c|}
\hline \multicolumn{7}{|c|}{ Coeficientes $^{\mathrm{a}}$} \\
\hline \multirow{2}{*}{\multicolumn{2}{|c|}{ Modelo }} & \multicolumn{2}{|c|}{$\begin{array}{l}\text { Coeficientes no } \\
\text { estandarizados }\end{array}$} & Coeficientes & $t$ & Sig. \\
\hline & & B & Error típ. & Beta & & \\
\hline \multirow{3}{*}{1} & (Constante) & 1,161 & 209 & & 5,569 &, 000 \\
\hline & Satisfacción General & 024 &, 005 & ,466 & 5,106 &, 000 \\
\hline & (Constante) &,- 256 &, 362 & &,- 706 & ,482 \\
\hline \multirow[t]{2}{*}{2} & Satisfacción General & 015 &, 005 & ,294 & 3,228 &, 002 \\
\hline & Índice de Clima General & ,663 & 145 & ,417 & 4,587 &, 000 \\
\hline
\end{tabular}

a. Variable dependiente: Índice de Desempeño en General

Análisis de la Satisfacción Laboral y el Clima Organizacional General como predictores de las dimensiones del desempeño.

\section{Dimensión Rendimiento y Productividad.}

Sólo la satisfacción laboral es un predictor significativo de esta dimensión, explicando un $46.6 \%$ de la variabilidad del rendimiento y productividad $(F(1 ; 94)=82.051 ; p<0.05) . \mathrm{Al}$ analizar el poder predictivo de la satisfacción sobre el rendimiento y productividad se observa una relación significativa y positiva entre estas variables (Ver tabla 2), de modo que al aumentar la satisfacción laboral, aumentará el Rendimiento y Productividad de los trabajadores $(\beta=0.683 ; t(95)=9.058$; $p<0.05)$. 
TABLA 2.

Coeficientes de satisfacción general como predictor de rendimiento y productividad.

\begin{tabular}{|c|c|c|c|c|c|c|}
\hline \multicolumn{7}{|c|}{ Coeficientes $^{a}$} \\
\hline & \multirow{3}{*}{ Modelo } & \multirow{2}{*}{\multicolumn{2}{|c|}{$\begin{array}{l}\text { Coeficientes no } \\
\text { estandarizados }\end{array}$}} & \multirow{2}{*}{$\begin{array}{l}\text { Coeficientes } \\
\text { estandarizados }\end{array}$} & \multirow[t]{2}{*}{$t$} & \multirow[t]{2}{*}{ Sig. } \\
\hline & & & & & & \\
\hline & & B & Error típ. & Beta & & \\
\hline \multirow[t]{3}{*}{1} & (Constante) & 167 & 243 & & ,684 & ,496 \\
\hline & Satisfacción & ,050 & ,006 & 683 & 9,058 &, 000 \\
\hline & General & & & & & \\
\hline
\end{tabular}

a. Variable dependiente: Rendimiento y Productividad Recategorizada

\section{Dimensión Condiciones Personales.}

Sólo el clima organizacional resultó ser un predictor significativo de esta dimensión, explicando un $19.4 \%$ de su variabilidad $(F(1$; $94)=23.905 ; p<0.05)$. Los coeficientes muestran que hay una relación significativa y positiva entre el clima y las condiciones personales $(\beta=0.450 ; t(95)=4.889 ; p<0.05)$ (Ver tabla 3).

TABLA 3.

Coeficientes clima general como predictor de la dimensión condiciones personales.

\begin{tabular}{|c|c|c|c|c|c|c|}
\hline \multicolumn{7}{|c|}{ Coeficientes $^{a}$} \\
\hline & \multirow{3}{*}{ Modelo } & \multirow{2}{*}{\multicolumn{2}{|c|}{$\begin{array}{l}\text { Coeficientes no } \\
\text { estandarizados }\end{array}$}} & \multirow{3}{*}{$\begin{array}{c}\text { Coeficientes } \\
\text { estandarizados } \\
\text { Beta }\end{array}$} & \multirow[t]{3}{*}{$t$} & \multirow[t]{3}{*}{ Sig. } \\
\hline & & & & & & \\
\hline & & $B$ & Error típ. & & & \\
\hline \multirow{2}{*}{1} & (Constante) &,- 333 &, 517 & &,- 645 &, 521 \\
\hline & Indice de Clima General & ,925 & 189 & ,450 & 4,889 &, 000 \\
\hline
\end{tabular}

a. Variable dependiente: Condiciones Personales Recategorizada

Dimensión Comportamiento del Funcionario

Sólo el clima organizacional resultó ser un predictor estadísticamente significativo, explicando un $18 \%$ de la variabilidad de dicha dimensión $(F(1 ; 94)=21.809 ; p<0.05)$.

TABLA 4.

Coeficientes clima general como predictor de la dimensión condiciones personales.

\begin{tabular}{|c|c|c|c|c|c|c|}
\hline \multicolumn{7}{|c|}{ Coeficientesa } \\
\hline & \multirow{3}{*}{ Modelo } & \multirow{2}{*}{\multicolumn{2}{|c|}{$\begin{array}{l}\text { Coeficientes no } \\
\text { estandarizados }\end{array}$}} & \multirow{3}{*}{$\begin{array}{c}\text { Coeficientes } \\
\text { estandarizados } \\
\text { Beta }\end{array}$} & \multirow[t]{3}{*}{$\mathrm{t}$} & \multirow[t]{3}{*}{ Sig. } \\
\hline & & & & & & \\
\hline & & B & Error típ. & & & \\
\hline \multirow[t]{2}{*}{1} & (Constante) &,- 600 & ,583 & & $-1,029$ & ,306 \\
\hline & Indice de Clima General & ,996 & ,213 & ,434 & 4,670 &, 000 \\
\hline
\end{tabular}

a. Variable dependiente: Comportamiento del Funcionario Recategorizada significativa y positiva, de modo que cuando mejora el clima organizacional general, mejora el comportamiento funcionario $(\beta=$ $0.434 ; \mathrm{t}(95)=4.670 ; p<0.05)($ Ver tabla 4$)$. 
En resumen, al considerar las dimensiones del desempeño, la Satisfacción general resulta ser un predictor significativo del Rendimiento y Productividad, mientras que el clima general resulta ser un predictor significativo de las dimensiones Condiciones Personales y Comportamiento del Funcionario.

\section{DISCUSIÓN}

Respecto a la relación entre las variables de interés, los resultados evidencian que existe una relación positiva y significativa entre el clima organizacional general, y la satisfacción laboral general, lo que lleva a aceptar la primera hipótesis de investigación. Esto es consecuente con los autores e investigaciones que reportan la existencia a través del tiempo de una relación significativa entre las variables (Salinas, Laguna \& Mendoza, 1994; Salgado, Remeseiro \& Iglesias, 1996; Silva, 1996; Parker et al., 2003; Chiang, 2004, Patterson, Warr \& West, 2004; Schulte, Ostroff, \& Kinicki, 2006). Evidentemente, dado que esta investigación es de tipo correlacional, sólo es posible hablar de una relación entre las variables, sin establecer un sentido de causalidad, es decir, se puede afirmar que mientras mejor sea la percepción del clima general en la organización, mayor es el grado de satisfacción de los miembros de la organización con el trabajo en general, 0 viceversa. Las dimensiones específicas del clima organizacional que correlacionaron de forma significativa y positiva con la satisfacción laboral general son: identidad, calidez, apoyo, responsabilidad, normas, recompensa y estructura. Estos resultados son coherentes con los meta-análisis reportados por Schratz (1993), en los que se señala que dimensiones como apoyo de supervisión, relaciones de compañerismo (calidez), responsabilidad (autonomía), recompensas, son algunas de las principales dimensiones de clima asociadas históricamente a la satisfacción. Sin embargo, en este estudio dimensiones como riesgo no resultaron asociarse significativamente a la satisfacción laboral, mientras que en investigaciones anteriores se han asociado significativamente a la satisfacción laboral. Esto puede deberse a que cada contexto organizacional es diferente, por lo que es posible encontrar discrepancias entre las distintas realidades o contextos laborales. Respecto a las dimensiones de satisfacción laboral (JDI), que se correlacionaron de manera significativa y positiva con el clima organizacional general se encuentran: satisfacción con la tarea actual; luego siguen las dimensiones satisfacción con la supervisión; compañeros de trabajo; oportunidades de ascenso y sueldo. La información concuerda en gran parte con lo reportado por Schratz (1993), ya que todas estas dimensiones, sumadas a la satisfacción laboral general, han sido históricamente las dimensiones más asociadas al clima organizacional, por lo que tal como señala este autor, las actitudes positivas hacia estos aspectos del trabajo, se relacionan con la percepción de un ambiente grato de trabajo.

La segunda hipótesis también se cumple, pues se encontró una relación significativa (y además positiva) entre el clima organizacional y el desempeño de los trabajadores, aunque el grado de asociación entre las variables es de carácter moderado. El desempeño general mostró una correlación significativa con el clima general y con ocho dimensiones de éste. El desempeño tuvo una mayor asociación con la dimensión de identidad, luego recompensa, apoyo, calidez, normas, conflicto, estructura y riesgo. Cabe destacar que los son congruentes con otros estudios en que se ha mostrado una relación entre el clima y desempeño, desde Forehand y Gilmer en 1964, hasta estudios más actuales (Kangis \& Williams, 2000; Fey \& Beamish, 2001; Burton \& Lauridsen, 2004; Griffith, 2006). Además los resultados concuerdan con los meta-análisis realizados por Schratz (1993), donde se menciona que 
dimensiones de clima como, apoyo, riesgo y decisiones, recompensas, relaciones entre compañeros y estructura se han asociado históricamente con desempeños y resultados organizacionales. Silva (1996), y Chiang (2004), han enfatizado que el clima organizacional puede ser una herramienta útil para mejorar el desempeño organizacional, ya que la evidencia indica que al mejorar la percepción de los elementos del clima mencionados anteriormente, aumentaría el desempeño de los trabajadores. Esto puede ser una explicación para la relación entre las variables encontrada en este estudio. La tercera hipótesis también se cumple, pues se encontró una relación significativa (y positiva), entre la satisfacción laboral y el desempeño de los trabajadores. El desempeño correlacionó significativamente con la satisfacción general y con cuatro de las dimensiones de ésta. La dimensión de satisfacción que tiene mayor asociación con el desempeño general es tarea en el trabajo actual, luego vienen compañeros de trabajo, supervisión y sueldo. Esto es consecuente con las investigaciones que han encontrado una relación positiva y significativa entre la satisfacción laboral y el desempeño, de tal forma que las actitudes positivas hacia el trabajo influyen en el buen desempeño (Kopelman, Brief \& Guzzo, 1990; Salinas, Laguna \& Mendoza, 1994; Salgado, Remeseiro \& Iglesias, 1996; Parker et al., 2003; Chiang, 2004; Patterson, Warr \& West, 2004; Griffith, 2006). La cuarta hipótesis no se cumple, pues el clima organizacional resultó ser mejor predictor del desempeño que la satisfacción laboral. Respecto a estos resultados surgen al menos cuatro posibles explicaciones. La primera se centra en la idea de que la medida de clima involucra dimensiones conductuales que no necesariamente se asocian al concepto de usualmente empleado (más similar al de productividad). Por otro lado la relación entre el clima y otras variables puede variar en función de las características de la muestra (Silva, 1996). Por lo tanto, la relación encontrada entre el clima, la satisfacción y el desempeño en esta empresa, puede variar en otros contextos organizacionales. Pero esta posible explicación debe tomarse con precaución, ya que los estudios metaanalíticos revelan ciertas correlaciones significativas entre el clima y el desempeño, y entre la satisfacción y el desempeño, por lo que es esperable encontrar una relación variable. La segunda explicación tiene que ver con la forma cómo se operacionalizan las variables, en este caso las medidas utilizadas para el desempeño, son de carácter subjetivo (evaluación de desempeño), no desde un enfoque económico. Generalmente los estudios que han mostrado un rol mediador de la satisfacción entre clima y desempeño, han tomado el desempeño desde un enfoque económico, como sinónimo de productividad, ganancias, etc. (Kopelman, Brief \& Guzzo, 1990; Parker et al., 2003; Patterson, Warr \& West, 2004). Lo anterior conduce también a la tercera posible explicación, para ello es relevante mencionar primero los resultados obtenidos al analizar el poder predictivo del clima y la satisfacción sobre las dimensiones específicas del desempeño (pues era probable también que el clima se relacionara con ciertos aspectos del desempeño y la satisfacción con otros, y que por eso ambas variables juntas sean predictores significativos del desempeño). Sólo la satisfacción laboral resultó ser un predictor significativo del rendimiento y la productividad, teniendo una relación significativa y positiva con esta dimensión. Esta información es congruente con los estudios en que se ha encontrado una mediación de la satisfacción sobre el clima y desempeño, 0 un efecto directo de la satisfacción sobre el desempeño, éste último ha sido medido a través de la productividad (calidad de producción, eficiente utilización de bienes y servicios), 0 de variables que son de corte más bien económico (Patterson, Warr \& West, 2004). Sólo el Clima Organizacional resulta ser un predictor significativo de las dimensiones de 
desempeño condiciones personales y comportamiento funcionario, teniendo una relación significativa y positiva con ambas variables. Aparte de la explicación de que la satisfacción laboral se ha asociado más con el desempeño económico, es posible que el clima se relacione más con las dimensiones comportamiento funcionario y condiciones personales, ya que el comportamiento del funcionario se relaciona con el cumplimiento de normas, deberes, misiones, en general aspectos de la estructura de trabajo, que generalmente son considerados en la medición del clima (dimensión estructura), pues hay teóricos como Litwin \& Stringer (1968), o Lawler, Hall \& Oldman (1974), que han evidenciado que el clima tendría una relación directa con la estructura organizacional, y la satisfacción no, necesitando ésta de variables mediadoras como el clima para relacionarse con variables estructurales (teniendo una relación indirecta con la estructura organizacional). Por otro lado la dimensión del desempeño condiciones personales, se relaciona con la forma de atender al usuario, relaciones interpersonales en el trabajo, comunicación, etc. Patterson et al. (2005), ha evidenciado al respecto que la percepción adecuada de la comunicación y relaciones interpersonales en el trabajo influyen en un buen clima organizacional, favoreciendo un buen desempeño, por ende si el desempeño es medido a través de dimensiones de este tipo (comportamiento funcionario mediante aspectos estructurales como cumplir normas y misiones, y condiciones personales mediante aspectos como los interpersonales), es probable que la percepción adecuada del clima favoreciera el desempeño en esas dimensiones. En concreto, se debe tener precaución en especificar bien el concepto de desempeño que se utilizará en un estudio determinado, sin generalizar los resultados que se obtengan, pues la medida que se utilice de desempeño sea económica o no, puede provocar un efecto diferenciador en la predicción que el clima y la satisfacción tengan respecto a esta variable. En resumen, la relación encontrada entre clima, satisfacción y desempeño en la empresa, es que tanto el clima organizacional como la satisfacción laboral son predictores significativos del desempeño organizacional, sin embargo el clima predice y se relaciona de forma positiva y significativa con aspectos normativos (comportamiento funcionario), y condiciones personales del desempeño, mientras que la satisfacción con el rendimiento y productividad. Estos resultados encontrados en un servicio público nacional, pueden ser un aporte para comenzar a aclarar la relación entre las variables, ya que los estudios o modelos que describen la relación del clima y satisfacción con aspectos específicos del desempeño, lo han medido más bien económica y unidimensionalmente (desempeño general, porcentaje de ganancias, etc).

Dentro de las limitaciones de esta investigación es que la muestra no es de gran tamaño, por lo que no es posible generalizar a partir de ésta, ni extrapolar los resultados a múltiples contextos organizacionales (ya sean de carácter público o privado), por ende, mientras más estudios existan sobre la relación entre estas tres variables, mayor evidencia empírica se tendrá de esta relación, y se aportará al mismo tiempo valiosa información para estudios meta-analíticos posteriores. A nivel local los trabajos de Chiang consideran avances importantes al respecto (Chiang, Salazar, Huerta, Núñez, 2008). Sin embargo, es más relevante aún aportar con nuevos conocimientos en esta área y que estén más cercanos a establecer causalidad entre las variables, por lo que se sugiere replicar este estudio en muestras más grandes, que permitan realizar análisis más finos de la relación de las variables, como los modelos de ecuaciones estructurales, que son un paso previo a los estudios causales, pues permiten ver más finamente la relación entre las variables, por ejemplo permitirían ver si existe mediación 
del clima sobre la satisfacción y las condiciones personales y comportamiento funcionario. Por otra parte, se puede mencionar como limitación del estudio la medida de desempeño utilizada, pues es una medida subjetiva en la cual sólo una persona (supervisor inmediato), evalúa el desempeño de cada funcionario, y por lo tanto no necesariamente da cuenta del desempeño real de los funcionarios de la empresa. Los servicios públicos en general suelen utilizar medidas muy subjetivas de evaluación de desempeño, en las cuales, las calificaciones o puntajes obtenidos por los funcionarios suelen ser demasiado elevados. Por todo lo anterior, se sugiere para futuras investigaciones utilizar medidas de desempeño más objetivas 0 validadas empíricamente. Así también, se podría utilizar como técnica de evaluación, un modelo integral de feedback en $360^{\circ}$, ya que otorgaría más objetividad al proceso, permitiendo que el sujeto no sólo sea evaluado por el supervisor, sino también por sus compañeros de trabajo, sus subordinados o por los clientes o usuarios de la organización. Otras medidas que se podrían utilizar al evaluar indicadores de desempeño en empresas públicas son por ejemplo las que mencionan Kangis \& Williams (2000), como los índices de productividad, ganancias de la empresa, rendimiento en base al capital empleado, $u$ otros indicadores objetivos de eficiencia y eficacia que generalmente son utilizados por las organizaciones. Dentro de las sugerencias para futuras líneas de investigación en el área, cabe señalar que como hoy en día se sabe sobre la existencia de relación entre variables de clima organizacional y satisfacción laboral, y que ambos son constructos distintos (clima perceptual), satisfacción (afectivo), es conveniente que sigan existiendo estudios que analicen separadamente clima y satisfacción y su efecto en variables de resultado (rendimiento, absentismo, rotación, accidentes, etc.), con el fin de seguir aclarando el efecto diferencial que pueda tener el clima y satisfacción en dimensiones de resultado o del desempeño.

También se recomienda realizar estudios de tipo longitudinal para medir la relación entre las variables en varios momentos en el tiempo de manera sistemática, y sin menospreciar el aporte que estudios bifocales utilizando no sólo metodología cualitativa sino también cuantitativa pueden entregar. No es recomendable realizar sólo estudios cualitativos de la relación entre estas variables (de hecho históricamente los estudios de esta relación han sido de carácter cuantitativo), pero sí estos estudios son un aporte, pues por ejemplo, nos dan una orientación de las dimensiones más relevantes a medir, por lo que construir instrumentos específicos de clima, y satisfacción para las realidades organizacionales a partir de resultados cualitativos, también es recomendable para realizar un mejor análisis de las variables. Este último punto se relaciona además con el hecho de que la interpretación del clima organizacional y los resultados que se obtengan, evidentemente dependen mucho de las dimensiones del clima que se incluyen en los estudios. Si bien en este estudio, se utilizaron cuestionarios generales empíricamente validados y confiables, cuyas dimensiones se han identificado como relevantes en la literatura, otras dimensiones $u$ otras medidas de dimensiones semejantes podrían cambiar en parte los resultados y su interpretación, por lo que en investigaciones futuras se necesita considerar diferentes dimensiones del clima organizacional y la satisfacción laboral. Para futuros estudios en empresas públicas 0 privadas también se pueden considerar aspectos tecnológicos y de infraestructura que podrían influir en el desempeño, pues pueden ayudar a entender mejor la relación entre clima y desempeño. Esto porque, muchas veces en las empresas públicas existen sistemas burocráticos que impiden contar con los recursos e infraestructura necesarios, por lo que si se percibe esta carencia, repercutirá 
en alcanzar un óptimo desempeño. Se debe considerar por ejemplo, que Fredericksen en sus estudios realizados en 1966, concluye que la predictibilidad del desempeño es más alta en un clima innovador que en uno altamente estructurado o burocrático y orientado a las normas (Chiang, 2004). Los modelos de desarrollo organizacional, no deben conformarse con una visión única, parcial o basarse en una sola dimensión del clima, sino que deben fundarse en un enfoque integral y oolítico. Por esto, replicar este tipo de estudios considerando además variables como la motivación laboral, compromiso organizacional, etc. (considerados en el modelo de Parker et al., 2003), puede ayudar a explicar mejor la relación entre las variables, y aumentar el porcentaje de variabilidad explicado del desempeño, pues estas variables también se han asociado significativamente con el desempeño. También cabe señalar que la medición del clima puede hacerse desde distintos niveles de análisis tomando no sólo la organización como objeto de análisis sino también al individuo, grupo o unidad funcional, como señala Peiró (1985), no se debe perder de vista la complejidad del mundo organizacional y que la subjetivación del ambiente organizacional se da a nivel individual, aunque con influencias grupales y que el entorno es una característica similar para muchos miembros, aunque con elementos diferenciales para los diferentes departamentos. Por lo que también considerar variables como nivel jerárquico, unidad de trabajo u otras variables organizacionales específicas al estudiar las relaciones entre clima, satisfacción y desempeño puede ser un aporte.

Finalmente, se debe señalar que este estudio podría ser un aporte, en el sentido de incluir otras dimensiones del desempeño además de la productividad, logrando establecer un efecto diferenciador en cuanto a los aspectos del desempeño que se pueden predecir ya sea por el clima organizacional o por la satisfacción laboral.

\section{REFERENCIAS}

Alcover, C., Martínez, D., \& Rodríguez, F. (2004). Introducción a la psicología del trabajo. España: Mcgraw-Hill.

Alfaro, A., \& Vecino, J. (1999). Medición de la satisfacción laboral mediante el cuestionario de satisfacción de necesidades de Porter. Revista de Psicología de Trabajo y las Organizaciones, 15 (1), 63-75.

Boada, J., Vallejo, R., \& Agulló, E. (2004). El burnout $y$ las manifestaciones psicosomáticas como consecuentes del clima organizacional y la satisfacción laboral. Psicothema, 16 (1), 125-131.

Brutus, S., \& Gorriti, M. (2005). La Evaluación Multifuente Feedback $360^{\circ}$. Revista de Psicología del Trabajo y de las Organizaciones, 21 (3), 235-252.

Burton, R., \& Lauridsen, J. (2004). The impact of organizational climate and strategic fit on firm performance. Human Resource Management, 43 (1), 67-82.

Carr, J.Z., Schmidt, A.M., Ford, J.K. \& DeShon, R.P. Climate perceptions matter: A meta-analytic path analysis relating molar climate, cognitive and affective states, and individual level work outcomes. Journal of Applied Psychology, 88, 605-619

Castro, C., \& Paredes, J. (2006). Estudio de validación de los instrumentos JDI y JIG en los trabajadores de Salud Primaria de la Región del Maule. Tesis (Psicólogo social y de las organizaciones). Talca, Chile, Universidad de Talca, Facultad de Psicología 2006.

Chiang, M. (2004). Relación entre Clima Organizacional y Satisfacción Laboral en grupos de profesores y/o investigadores universitarios. Tesis (Doctorado en Gestión de Recursos Humanos). Madrid, España, Universidad de Málaga, Departamento de Gestión empresarial 2004.

Chiang, M., Salazar, M., Huerta, P. y Núñez, A. Clima organizacional y satisfacción 
laboral en organizaciones del sector estatal (instituciones públicas). Desarrollo, adaptación y validación de instrumentos. Universum, 23 (2), 66-85

Cuadra, A. \& Veloso, C. Liderazgo, Clima y Satisfacción Laboral en las Organizaciones. Revista Universum, 22, 40-56

Davis, K., \& Newstrom, J. (1999). Comportamiento humano en el trabajo. México: McGraw-Hill.

De Juan, P. (2004). La "P" mayúscula de los procesos de selección. Revista de Psicología del Trabajo y de las Organizaciones, 20 (2), 263-271.

Fey, C., \& Beamish, P. (2001). Organizational Climate and Similarity and Performance: International Joint Ventures in Russia. Organization Studies, 22 (5), 853-882.

Furnham, A. (2001). Psicología organizacional: el comportamiento del individuo en las organizaciones. México: Oxford University.

Gamero, C. (2003). Análisis económico de la satisfacción laboral. Tesis (Doctorado en Estadística y Econometría). Madrid, España, Universidad de Málaga, Departamento de Economía Aplicada. 2003. Extraído el 20 de Mayo de 2007 desde: http://www.eumed.net/tesis/cgb/index.h tm.

Gómez, C. (2004). Diseño, construcción y validación de un instrumento que evalúa clima organizacional en empresas colombianas, desde la teoría de respuesta al ítem. Acta Colombiana de Psicología, 11, 97-113

Griffith, J. (2006). A Compositional Analysis of the Organizational ClimatePerformance Relation: Public Schools as Organizations. Journal of Applied Social Psychology, 36 (8), 1848-1880.

Imran, R., Saeed, T., Anis-Ul-Haq, M. \& Fatima, A. (2010). Organizational climate as a predictor of innovative work behavior. African Journal of Business Management, 15, 3337-3343
Ironson, G. Brannick, M. Smith, R. Gibson, W. Paul, K. (1989). Construction of a Job in General Scale: A Comparison of Global, Composite, and Specific Measures. Journal of Applied Psychology, 74 (2). 193-200.

Kangis, P., \& Williams, D. (2000). Organisational Climate and corporate performance: an empirical investigation. Management Decision, 38 (8), 531-540.

Kopelman, R., Brief, A., \& Guzzo, A. (1990). The role of climate and culture in productivity. En B. Schneider (Ed.), Organizational Climate and Culture, 282-318. San Francisco: Josef-Bass.

Lawler, E., Hall, D., \& Oldman, G. (1974). Organizational climate: Relationship to organizational structure, process, and performance. Organizational Behavior Human Performance, 11, 139-155.

Litwin, G. \& Stringer, R. (1968), Motivation and Organizational Climate. Boston: Harvard University Press.

Martin, K., \& Cullen, J. (2006). Continuities and extensions of ethical climate theory: a meta analytic review. Journal of Business Ethics, 69, 175-194.

Parker, C., Baltes, B., Young, S., Huff, J., Altmann, R., Lacost, H., \& Roberts, J. (2003). Relationships between psychological climate perceptions and work outcomes a meta-analytic review. Journal of Organizational Behavior, 24, 389-416.

Patterson, M., Warr, P., \& West, M. (2004). Organizational climate and company productivity: the role of employee affect and employee level. Journal of Occupational and Organizational Psychology, 77, 193-216.

Patterson, M., West, M., Shackleton, V., Dawson, J., Lawthom, R., Maitlis, S., Robinson, D., \& Wallace, A. (2005). Validating the organizational climate measure: links to managerial practices, productivity and innovation. Journal of Organizacional Behavior, 26, 379-408. 
Peiró, J. (1985). Evaluación psicológica de las organizaciones. Evaluación Psicológica, 1 (2), 189-239.

Peraza, Y., \& García, M. (2004). Clima organizacional: concepto y experiencias. Revista transporte, desarrollo y medio ambiente, 21 (2), 27-30.

Pritchard, R. (1992). Organizational productivity. Handbook of industrial and organizational psychology 3, 443-471.

Reza, H., \& Jahandari, R. (2005). The Organizational Climate of Kerman Shahid Bahonar University. Public Personel Management, 34 (3), 247260.

Robbins, S. (2001). Comportamiento organizacional: conceptos, controversias y aplicaciones. México: Prentince-Hall Hispanoamericana.

Rodríguez, D. (2004). Diagnóstico organizacional. Santiago, Chile: Universidad Católica de Chile.

Saari, L., \& Judge, T. (2004). Employee attitudes and job satisfaction. Human Resource Management, 43 (4), 395407.

Salgado, J., Remeseiro, C., \& Iglesias, M. (1996). Clima organizacional y satisfacción laboral en una Pyme. Psicohtema, 8 (2), 329-335.

Salgado, E. (2006). Dos textos: sobre el desempeño individual en las organizaciones. Monografias de Administración, 93, 1-54.

Salinas, C., Laguna, J., \& Mendoza, M. (1994). La satisfacción laboral y su papel en la evaluación de la calidad de la atención médica. Salud Pública, 36 (1), 22-29.

Schratz, M. (1993). Recommendatios for the measurement of organizational climate. Documento presentado en el Reunión anual de la American Psychological Association, Toronto, Ontario, Canada. Schulte, M., Ostroff, C., \& Kinicki, A. (2006). Organizational climate systems and psychological climate perceptions: A cross-level study of climate-satisfaction relation. Journal of Occupational and Organizational Psycohology, 79, 645671.

Silva, M. (1996). El Clima en las Organizaciones. Teoría, método e intervención. Barcelona: EUB, S.L.

Smith, P. Kendall, L. Hulin, C. (1969). The measurement of satisfaction in work and retirement. Chicago. Rand McNally.

Sziklai, G. (2006). Estudiar y gestionar el clima organizacional como medio para lograr una ventaja competitiva. Revista capacitación, 52. Extraído el 16 de Mayo de 2007 desde http://www.ccesff.cl/CCESFF/Revista/52/P524555657 6768687867rt.pdf.

\section{AGRADECIMIENTOS}

Los autores agradecen al Programa de Investigación en Calidad de Vida y Ambientes Saludables de la Facultad de Psicología de la Universidad de Talca. 